\title{
Quantitative Phase Retrieval by Ptychography in TEM
}

\author{
F. Hüe, ${ }^{*}$ A.M. Maiden, ${ }^{* *}$ J.M. Rodenburg, ${ }^{* *}$ and P.A. Midgley* \\ * Department of Materials Science and Metallurgy, University of Cambridge, Pembroke Street, \\ Cambridge, CB2 3QZ, UK. \\ ** Department of Electrical and Electronic Engineering, University of Sheffield, Sheffield, S13JD, \\ UK.
}

We present a new way to determine quantitative phase shift at the nanometer scale. The technique used, called ptychography [1], allows us to retrieve phase and amplitude of an object from its diffraction patterns. This method was originally proposed to overcome the problem of lens aberration by only recording the diffracted intensities in the far-field to improve spatial resolution [2]. Experimentally, a confined illumination function is moved laterally to several positions and we record diffraction patterns that contain varying sets of information about the object. The algorithm used is a Fienup-type algorithm but treats in parallel all the data for each position of the probe. To date, it has shown excellent results in light optics and x-ray experiments [3-4] and we now present here encouraging results for TEM applications.

Figure 1 shows a schematic representation of the experimental setup on a Tecnai FEGTEM. An overfocused probe scans the specimen $\left(\mathrm{Fe}_{30} \mathrm{Ni}_{70}\right.$ nanoparticles) and the diffraction patterns in the Fraunhofer plane are recorded on the CCD camera (Gatan 1024 x 1024 pixels) for 25 overlapping positions (the step between each position is $10 \mathrm{~nm}$ and the probe is $50 \mathrm{~nm}$ wide). We used a new version of the original Ptychographical Iterative Engine (PIE) which allows to retrieve either the probe and the object [5]. The feedback parameter is set to 1 to increase the speed of convergence. The new ePIE algorithm was running for 4000 iterations, updating the probe after 2000. The results are presented in Figure 2. In this case, the object phase is proportional to the mean inner potential (MIP) and the thickness of the specimen (no electric or magnetic field). Different profiles taken across the object show quantitative phase measurements. We compared these results with those from the more frequently used off-axis holography. Ptychography here is based on an in-line hologram, does not require a biprism and provides good results even with partial coherence. The spatial resolution is limited by the probe position accuracy, in our case $1 \mathrm{~nm}$. The phase resolution is limited mainly by the noise in the reconstruction and is estimated to be $0.2 \mathrm{rad}$.

\section{References}

[1] J. M. Rodenburg and R.H.T. Bates, Phil. Trans. R. Soc. Lond. A. 339 (1992) 521-553.

[2] P.D. Nellist et al, Nature 374 (1995) 630-632.

[3] J. M. Rodenburg, A. C. Hurst, and A. G. Cullis, Ultramicroscopy, 107 (2007) 227-231.

[4] J. M. Rodenburg et al., Phys. Rev. Lett., 98 (2007) 034801.

[5] A.M. Maiden and J.M. Rodenburg, Ultramicroscopy 109 (2009) 1256-1262.

[6] The author(s) wish to gratefully acknowledge the support of EPSRC for funding this work which was part of the - Basic Technology Grant (EP/E034055/1)- Ultimate Microscopy: wavelength limited resolution without high quality lenses. 


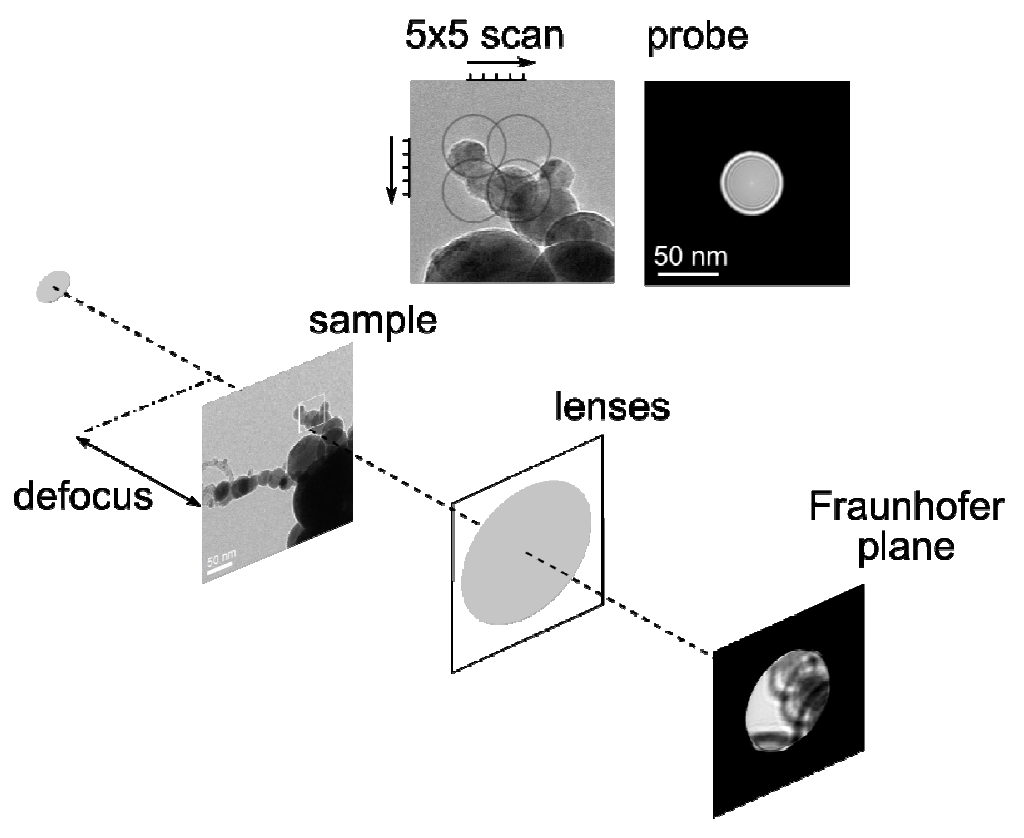

FIG. 1. Experimental setup for a shifting coherent electron probe on a specimen. The probe is $50 \mathrm{~nm}$ wide in the specimen plane and for each position of a 5 by 5 grid the corresponding diffraction pattern is recorded. An overlap occurs between each probe.

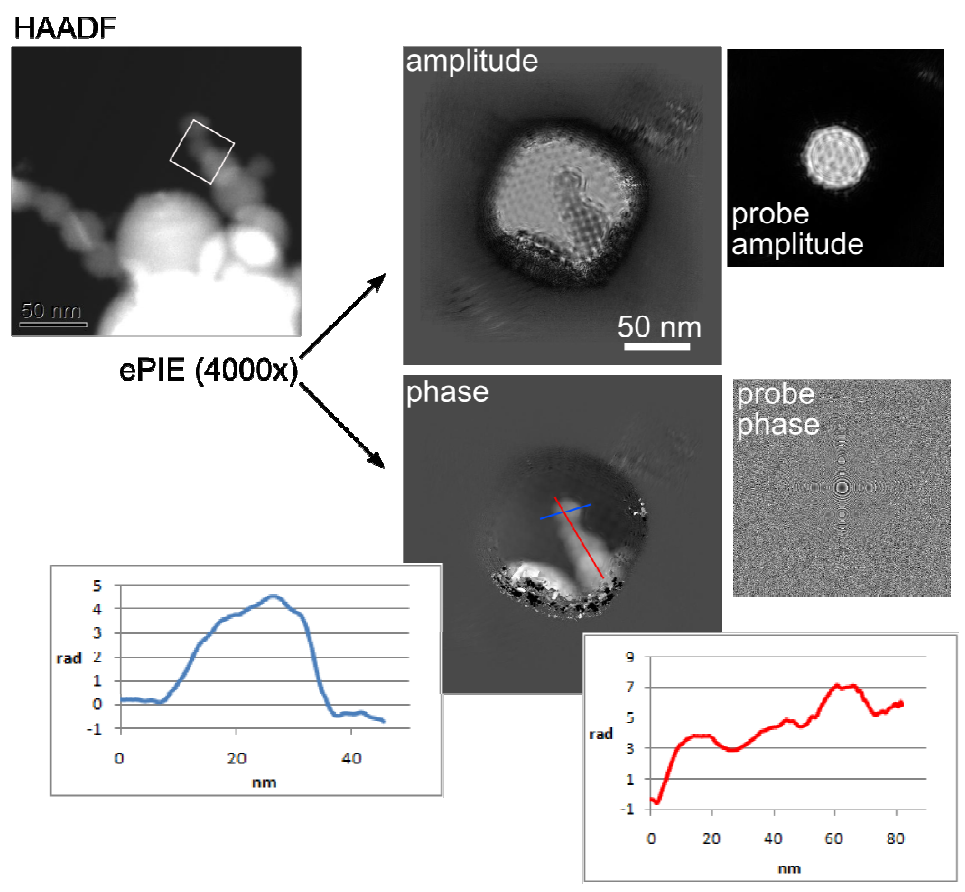

FIG. 2. Ptychographical reconstruction of the amplitude and the phase of $\mathrm{Fe}_{30} \mathrm{Ni}_{70}$ particles. The ePIEalgorithm was running for 4000 iterations with an update of the probe after 2000 iterations. The values of the phase shift are in good agreement with theory. 